\title{
MEDICAL ULTRASOUND USED FOR HYDRODYNAMICS STUDY AND ERODED SURFACE MAPPING IN PHYSICAL MODELING OF TURBIDITY CURRENTS
}

\author{
BOFFO, CAROLINA(1), PEREIRA, LUCAS(2), OLIVEIRA, TIAGO(3), BAYER DA SILVA, DANIEL ${ }^{(4)}$, BORGES, \\ ANA LUIZA(5), MANICA, RAFAEL ${ }^{(6)}$, MORAES, MARCO(7) \& PARAIZO, PAULO(8) \\ $(1,2,4,5,6)$ Density currents Research Center (NECOD) \\ Universidade Federal do Rio Grande do Sul - Brazil. \\ (carolinahb@gmail.com; freitas.pereira@ufrgs.br; daniel.bayer.silva@gmail.com, ana.borges@ufrgs.br; rafaelmanica@gmail.com) \\ $(3,7,8)$ Petrobras - Brazil. \\ (tiagoagne@petrobras.com.br; paraizo@petrobras.com.br)
}

\begin{abstract}
One of the great challenges in the physical modeling of turbidity currents is to acquire data with equipment that do not cause disturbance. Imaging equipment, such as video cameras, are an alternative for recording experiments without disturbances. However, for flows with high concentration, or where the material has unfavorable color (such as coal), visualization and analysis end up being impaired. An alternative for recording such flows is the application of an ultrasonic imaging equipment, which allows the acquisition of data with good quality and resolution. Experiments performed in NECOD laboratory (UFRGS - Brazil) showed that the images obtained by a medical ultrasound equipment could provide good qualitative and quantitative data. In a first application, the ultrasound images suggest that there is a relationship between the different current layers and the velocity and concentration profiles. For this study, the gray scale colors produced by the medical ultrasound equipment were used to calculate numerical values, by the Matlab tool with a new mathematical code. Such values allow obtaining spectra of density correlating them with the measured velocity (UVP) and concentration (UHCM) profiles. In a second experiment, the medical ultrasound enables to measure and map the evolution of the slope profile eroded by turbidity currents. An image sequence obtained during the experiment, registered a series of slope profiles allowed the mapping of the evolution and formation of a subaqueous canyon. The ultrasound is a good tool to acquire images from flow simulations, including for experiments produced in small scale.
\end{abstract}

Keywords: Turbidity currents, medical ultrasound, hydrodynamic visualization, surface mapping.

\section{INTRODUCTION}

The study of density or turbidity currents, which occur in the ocean floor, has been a focus of great interest in the last decades (Fildani, 2017). One reason to improve the understanding of these deep-water systems is to increase the efficiency of the exploration and production of petroleum resources (Weimer \& Slatt, 2004). As such, the need for a better understanding the genesis and characteristics of the deep-water sediments, especially those with potential as petroleum reservoirs, has increased the demand for research on turbidity currents, including a better characterization of sediment transport mechanisms and depositional processes (Kneller, 1995). Presently, many efforts are on the way to monitor the seafloor, in an effort for directly measure the attributes of such flows (Paull et al., 2003; Xu \& Noble, 2004, 2009; Xu et al., 2014). However, due to the large magnitude and infrequency of these events, an adequate record of these flows is a rarity (Talling et al., 2012).

The alternative to improve the understanding of natural turbidity currents is the use of integrated physical and numerical modeling (Meiburg \& Kneller, 2010; Sequeiros, 2012). Such integration allows to obtain data that could not be directly measured in natural environments. Numerical modeling demands high-processing capacity, the development of complex models with well-defined boundary conditions and a set of partial differential equations, along with adequate calibration and validation. Often, the data used for calibration and validation are produced by physical models. Physical modeling, on the other hand, is quite robust and is widely used for a long time to study density flows on reduced scales. It also has a vast application in engineering (Yalin, 1971).

The scale of the physical model defines the type of equipment (instrumentation) applied to acquire flow parameters. The experiment scale may limit the experimental model, in the case of sediment transport and deposition. In the study of sedimentary deposits using physical simulation, the sediment grain size is the main factor that limits the reality simplification and scale of the model; in this case, it is necessary to consider the effect of sediment grain size and sediment mass of the experiments (Paola et al., 2009). In the measurement of flow parameters, most of the equipment used to measure velocity and concentration are intrusive, causing perturbation in the flow, which can affect the quality of the obtained data. Profilers and sensors which operation principle is based on ultrasound (UVP, UHCM, ADV) have a limit for maximum concentration of suspended 
particles, which makes it almost impossible to investigate high-concentration flows. Such limitation was confirmed in our experiments.

An alternative to obtain hydrodynamic parameters in experimental flows is the use of imaging equipment, such as video cameras and high-speed photographic cameras. Using image sequential analysis and a specific software, spectrum maps of flow velocity and concentration can be produced (i.e., PIV). However, these techniques have some limitations, the main one being a limit in the concentration of suspended particles, in order to obtain satisfactory results.

The medical ultrasound has been used for a few decades in the medical area because it is a fast and nonintrusive method, and presents good acquisition results. Such equipment allows the observation and recognition of internal structures due to the detection of stratified layers of different densities; and responds in different frequencies of attenuation for the emitted signal (layers with different impedances). The time elapsed between the signal emission and its return (echo) is used to calculate the thicknesses of the stratified layers. The intensity of the sound received is translated into grayscale values, which is a function of the medium impedance. Increasing the wave frequency results in higher resolution for the generated images, but also increases the attenuation, decreasing the beam zone (McDicken \& Anderson, 2011; Chan \& Perlas, 2011).

This equipment is being used in our experiments for some time, mainly to characterize the geometries and flow structures, measuring the turbidity current dynamics and the resulting deposit characteristics (Brito et al., 2009; Baas et al., 2014). In the present work, our effort and main objective is to test the possibility of using the medical ultrasound to acquire additional qualitative and quantitative data for physical modeling experiments of turbidity currents.

\section{METHODOLOGY}

Two different experimental setups applying the medical ultrasound were considered. The first comprises experiments of turbidity currents simulated in a glass flume, in order to obtain hydrodynamic parameters of the flows. In a second approach, a series of turbidity currents, running down over a constructed slope in a basin tank, caused erosion and the formation of a subaqueous slope canyon, where the knickpoint migration could be traced. The following items present a summary of both experimental approaches.

\subsection{Physical modeling of turbidity currents}

The experiments were performed in a glass flume $15.0 \mathrm{~m}$ long, $0.4 \mathrm{~m}$ wide and $1.0 \mathrm{~m}$ deep, with a flat bottom, fed through a rectangular diffuser connected to a raised reservoir. In the experiments, we used mineral coal mixtures $\left(d_{10}=15 \mu \mathrm{m}, d_{50}=85 \mu \mathrm{m}\right.$ and $\left.d_{90}=250 \mu \mathrm{m}\right)$ with volumetric concentrations varying between 1.83 and $37.91 \%$, injecting volumes ranging from 0.2 to $0.4 \mathrm{~m}^{3}$, with a constant discharge of $50 \mathrm{~L}^{\mathrm{min}} \mathrm{m}^{-1}$. The flow parameters of these experiments were obtained using a medical ultrasound equipment (Siemens - GM6600A2A00, probe 7.5L40 - positioned at $4.4 \mathrm{~m}$ ), and profilers for the velocity (Ultrasound Doppler Velocity Profiling - Metflow $-2 \mathrm{MHz}$ transducer) and concentration (Ultrasonic High Concentration Meter - Deltares), positioned along the flume $6.4 \mathrm{~m}$ away from the diffuser. The arrangement and positioning of the velocity and concentration probes follow similar methodologies adopted by Best et al (2001), Felix et al (2005), Felix and Peakall (2006) and Manica (2009).

\subsection{Physical modeling of subaqueous slope erosion and canyon formation}

The experiment was carried out in a basin tank $2.5 \mathrm{~m}$ long, $2.0 \mathrm{~m}$ wide and $0.6 \mathrm{~m}$ deep, equipped with a maximum water-level control system and a bottom drain.

A semicircular sand deposit (1.7 m wide, $0.8 \mathrm{~m}$ long and $0.25 \mathrm{~m}$ thick) was built to represent an underwater shelf-edge and slope morphology. The sediment used in the construction of the deposit was mineral coal, with a coarse sand caliper $\left(d_{10}=422.12 \mu \mathrm{m}, d_{50}=631.11 \mu \mathrm{m}\right.$ and $\left.d_{90}=961.18 \mu \mathrm{m}\right)$. To model the turbidity currents, a hose was installed at the shallow (upper) part of the deposit (shelf region) to inject mixtures with different volumetric concentration of sediments (between 0.625 and $5.625 \%$ ), and the material used was fine silt limestone $\left(d_{10}=0.93 \mu \mathrm{m}, d_{50}=4.16 \mu \mathrm{m}\right.$ and $\left.d_{90}=22.19 \mu \mathrm{m}\right)$, with maximum discharge of $0.3 \mathrm{~L} \cdot \mathrm{min}^{-1}$. The medical ultrasound equipment was positioned over the emerging slope channel thalweg. The ultrasound images recorded the slope profile evolution as the turbidity currents run down the slope, also aiding bathymetric data acquisition.

\subsection{Data treatment}

The recorded video sections were analyzed for each individual turbidity current experiment, and an image treatment approach was developed to calculate average values for the grayscale to represent the ultrasound vertical density profiles over time. The medical ultrasound record area is $12 \times 3.5 \mathrm{~cm}$, corresponding to $350 \times 102$ pixels $(35,700$ pixels) with $343 \times 343 \mu \mathrm{m}$ of area each one (Fig. 1). The medical ultrasound pixel resolution has the same size of the mineral coal $\left(d_{90}=250 \mu \mathrm{m}\right)$ used in the experiments. In order to capture a wider area to ensure that a single particle do not occupy a whole pixel, an cell composed by 49 pixels (area with $7 \times 7$ pixels) was applied to establish average values for the intensity color grade $(2.27 \times 2.27 \mathrm{~mm}$ each one). Thus, for each ultrasound image was produced a vertical line of analysis, composed of 50 cells. The average 
values of intensity, for each cell, were converted into a grayscale with values between 0 to 256 . The output data corresponds to a matrix of time $(\mathrm{x})$, vertical position $(\mathrm{y})$ and average the intensity values $(\mathrm{z})$. A flow spectrum generated with this data permits a visual comparison with the velocity and concentration spectrum, in order to investigate if there is a relationship between the measured parameters.

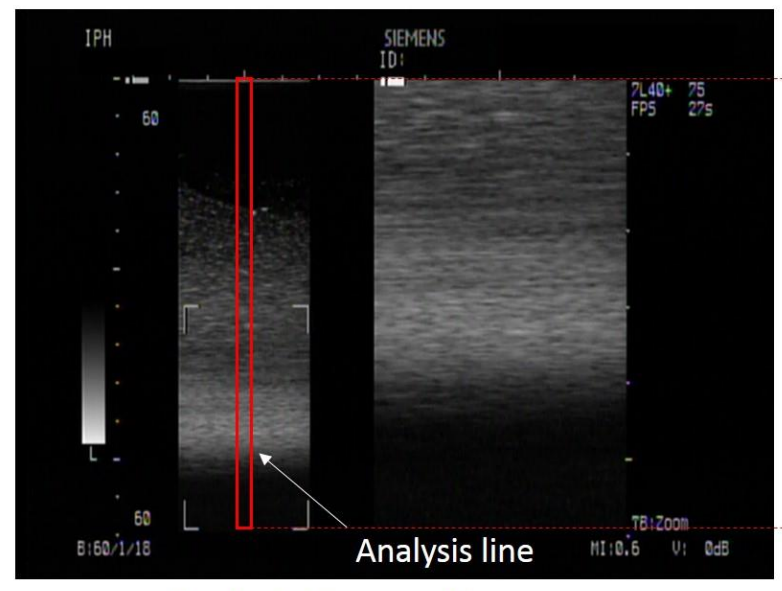

Medical ultrasound frame

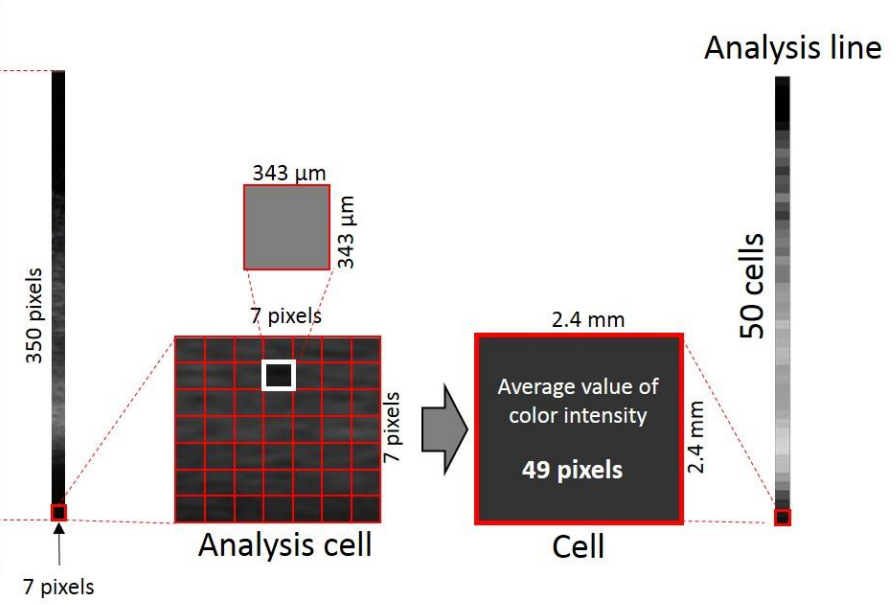

Figure 1. Illustrative image of the frame analysis, presenting an original high-resolution image window (left) converted into a line with average color intensity (right).

Considering that the spatial resolution of the velocity and concentration data does not have the same spatial resolution as the medical ultrasound data, those were compared adjusting the vertical profile measured. The profiles were adjusted according to the equations of Michon et al. (1955) and Altinakar (1988):

$$
\begin{gathered}
\frac{u(z)}{U_{m}}=\left(\frac{z}{h_{m}}\right)^{1 / n} \quad \text { for } z<h_{m} \\
\frac{u(z)}{U_{m}}=e^{\left[-\alpha\left(\frac{z-h_{m}}{h_{t}-h_{m}}\right)^{m}\right]} \text { for } z>h_{m}
\end{gathered}
$$

Where $u(z)$ denotes the vertical velocity, $h_{t}$ is the total height of the current, $U_{m}$ is the maximum velocity in the profile, $h_{m}$ is the height of occurrence of $U_{m}, z$ is the vertical distance from the bottom, and $n, \alpha$ and $m$ are the adjustment parameters.

The equation (3) was used to adjust the concentration profile, for which $C(z)$ denotes the volumetric vertical concentration, $C_{r}$ the maximum concentration in the profile and $A$ e $B$ are adjustment parameters. This treatment produced a matrix of velocity and concentration with the same size as that obtained by the medical ultrasound analysis.

$$
\frac{C(z)}{C_{r}}=A e^{\left(B \frac{z}{h_{t}}\right)}
$$

To map the erosional surface caused by the turbidity currents, the maximum measurement area permitted by the ultrasound equipment was acquired, corresponding to $12 \times 3.5 \mathrm{~cm}$. A careful analysis of each individual frame, in a sequence, allowed to trace the slope profile evolution through time. To establish a line that represents the bed bathymetry, a cell with $3 \times 3$ pixels was used to calculate the average grayscale intensity. The area with maximum value of intensity is used to identify the vertical position of the slope surface. The position of each cell produced a line with spatial coordinates $(x, y)$, highlighting the bed bathymetry.

The software Matlab ${ }^{\mathrm{TM}}$ was used to analyze and process the ultrasound, velocity and concentration datasets. The software Surfer ${ }^{\circledR}$ was applied to calculate the color grid for the intensity, velocity and concentration profiles.

\section{RESULTS}

Two different experimental setups were tested with the purpose of investigating the potential applications for the medical ultrasound to acquire additional qualitative and quantitative data. The results will be presented separately, further illustrating the full scope for this study. 


\subsection{Treatment of medical ultrasound data to flow analysis}

We present a discussion based on two experiments with volumetric concentrations of 12 and $40 \%$, both with injection of $200 \mathrm{~L}$ of mixture. The characteristics of each of the experiments were: $(A)$ the flow that reached the highest velocities was the one with lower concentration (12\%), reaching values up to $120 \mathrm{~mm} \cdot \mathrm{s}^{-1}$; (B) the flow with higher concentration (40\%) which exceeded the maximum concentration limit of the perfilometer had not effective velocity data registered. For each experiment, segments from the medical ultrasound videos were analyzed and selected to generate a flow concentration spectrum time series (Figure 2). The data window selected for this analysis represents to the full establishment of the flow evolution, circa $50 \mathrm{~s}$ after the head of the current pass through the sensors, and included the time of injection interruption. A total of 180 seconds window was consider for the analysis (Figure 2). Aiming to compare all the information, the velocity and concentration data registered with profilers were treated to correspond to the same time interval selected for the ultrasound data. However, it is important to consider that the profilers were positioned at a relative downstream points in relation to the ultrasound.

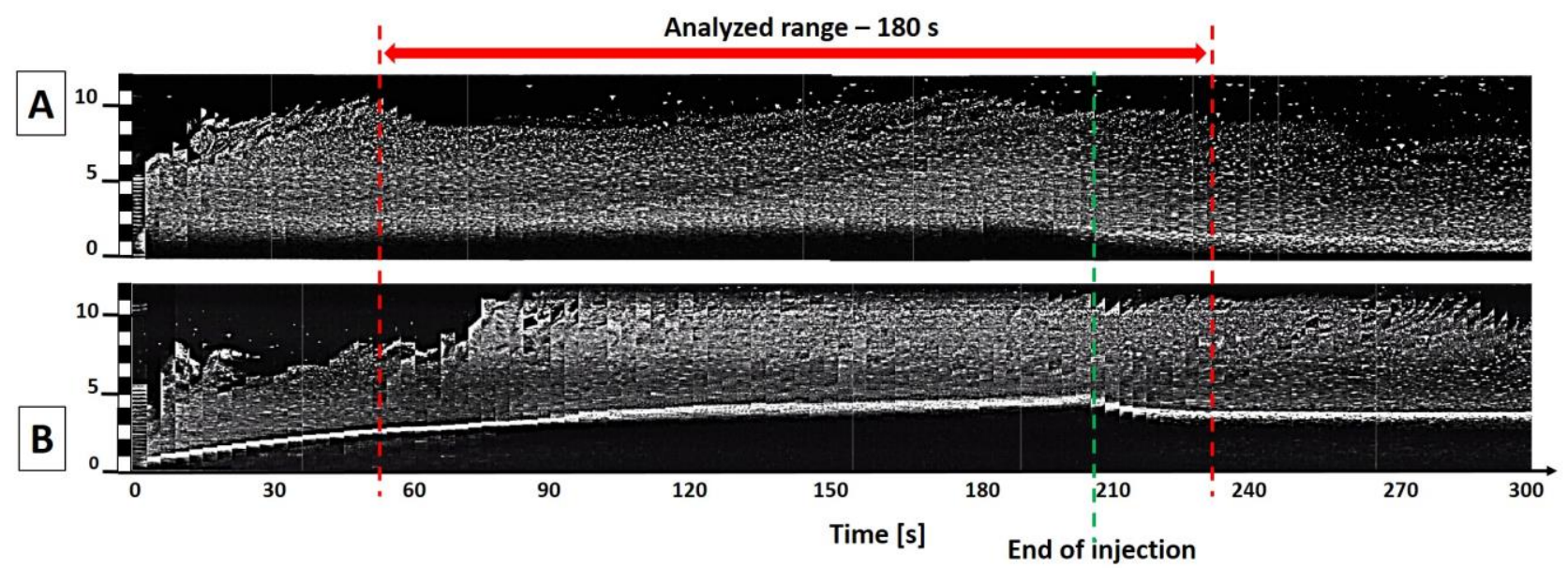

Figure 2. Flow spectrum image produced from the frame time series of the medical ultrasound acquisition. The images correspond to the experiments with volumetric concentrations of $12 \%$ (A) and $40 \%$ (B). The time zero $(T=0 \mathrm{~s})$ corresponds to the head of the turbidity current at the time of the passage through the ultrasound probe. The green line indicates the injection interruption and the red lines delimit the time range of data analyzed in this work.

The raw data from the medical ultrasound measurements (grayscale) is not clear for points with very close colors. To improve this information aiming to compare all the data available, we processed and converted the grayscale into a color scale of intensity (Figure 3), producing a color intensity spectrum for each experiment. This approach permitted to intensify the color variations, improving the interpretation and identification of the internal characteristics of the flows. The data treatment allowed the construction of matrices for the color intensity, concentration and velocity spectrum maps for each experiment. The medical ultrasound data analysis shows color intensity values between 0 and 160, and the highest apparent densities were recorded in the experiment mixture which had the highest concentration (40\%), as expected.

Through the visual analysis of the color intensity spectrum images, it is possible to identify the existence of layers with different colors (Figure 3). Such differences are interpreted as a response to variations in density. The mixing layer of the flows, i.e. their upper region, where there is an exchange between the current and the ambient fluid, appears to have lower densities. The density around the medical ultrasound sensor $(12 \mathrm{~cm}$ above the bottom) is close to the water density ( 0 to 20 in color intensity). The measured ultrasound density values are prone to increase towards the bottom.

From the medical ultrasound probe at the top, this trend is observed until about half of the current height (between 4 and $6 \mathrm{~cm}$ from the bottom). However, in the region near the flume bottom (between 0 and $3 \mathrm{~cm}$ ), which corresponds to the basal layer region of the current, the ultrasound data suggests the existence of a lowdensity layer. In the case of the high concentration flow (40\%), an abrupt density contrast occurs at this basal layer region (Fig. 3 - B). This is not well understood, as it was expected that the basal high concentration layer should be represented by high-density values. However, we consider that it is possibly related to limitations of the ultrasound probe to recognize and record information in conditions of very high concentration. 


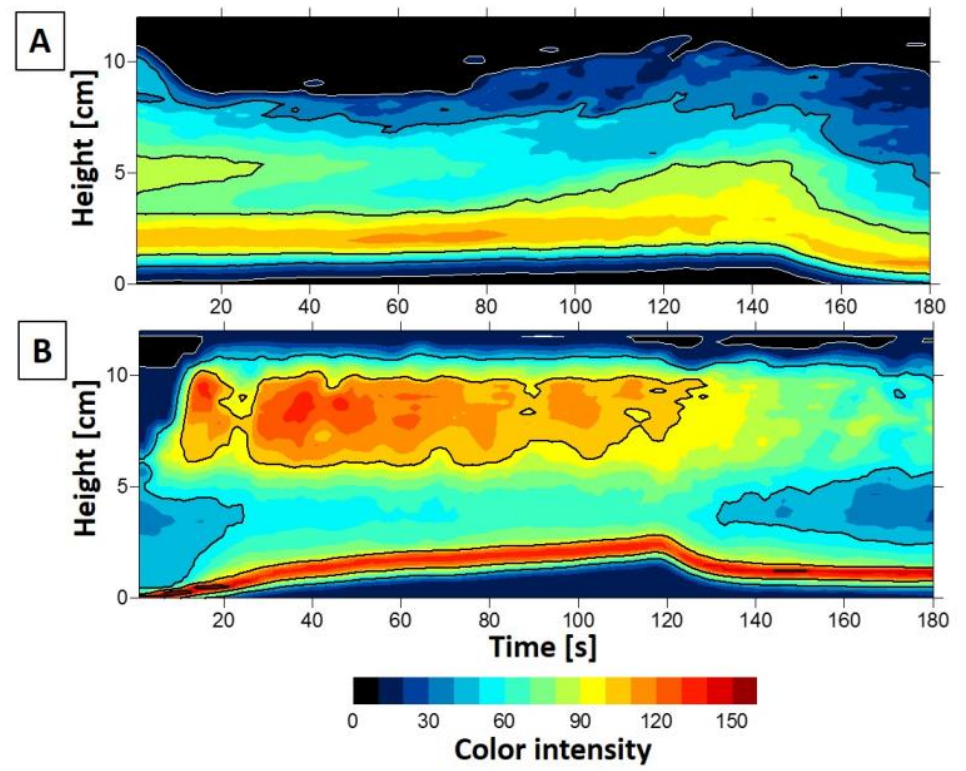

Figure 3. Ultrasound intensity spectrum variations obtained by the medical ultrasound videos data processing, to the experiments with $12 \%$ (A) and $40 \%$ (B) of volumetric concentrations. The cold colors correspond to lower densities (low intensity) and the hot colors correspond to higher densities (high density).

\subsection{Comparison of medical ultrasound data with velocity and concentration profiles}

On a preliminary visual comparison between the ultrasound color intensity with the velocity and concentration spectrum maps, a direct relationship is not easily recognized. Probably because the ultrasound color intensities are not dependent only on density.

On the other hand, when overlapping the color intensity curves, it is possible to identify similarities with the velocity and concentration spectrum maps. The concentration shows a fair correlation with the color intensities, and it is possible to identify a geometric similarity (Figure 4).
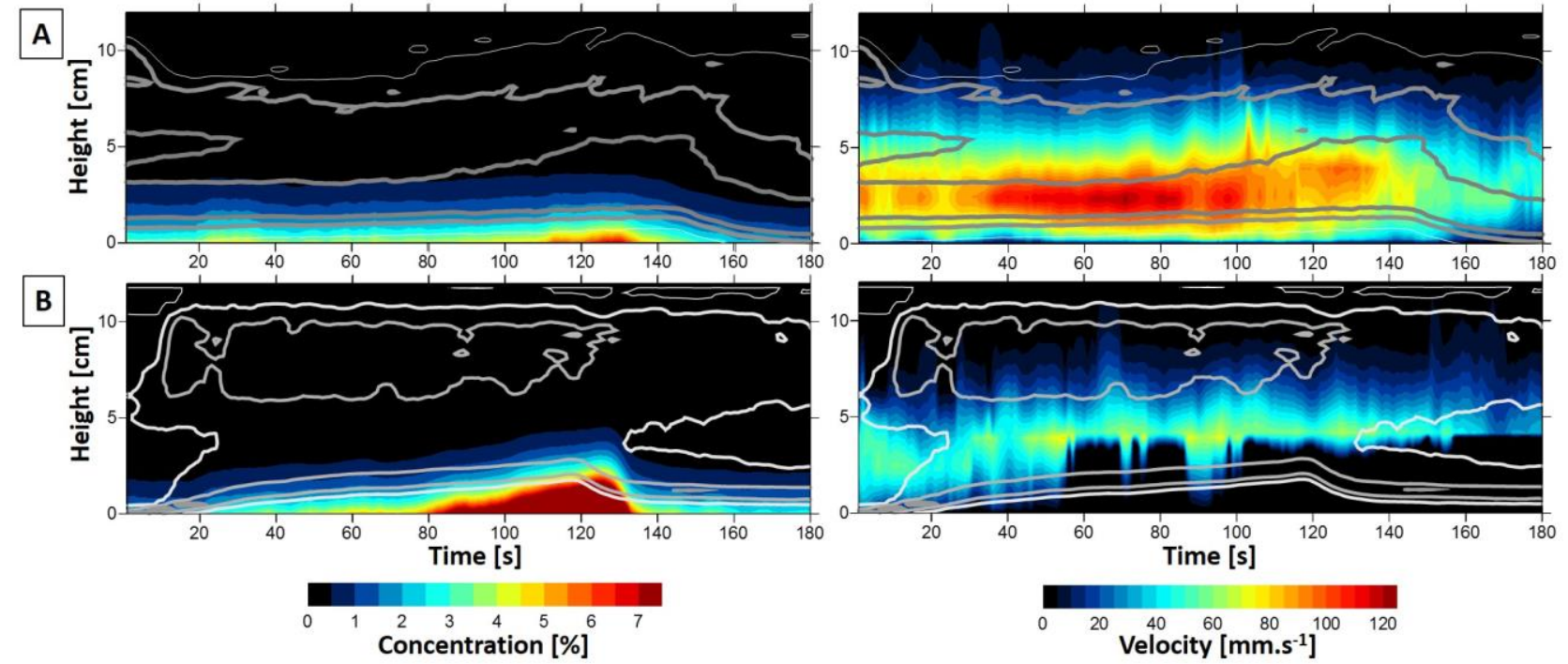

Figure 4. Concentration and velocity spectrum maps measured by the perfilometers. Experiments with volumetric concentrations of $12 \%(\mathrm{~A})$ and $40 \%$ (B). The cold colors correspond to regions of lower concentration (left column) and velocity (right column) and the hot colors to regions of higher concentration and velocity. The white lines plotted correspond to the ultrasound density curves from the maps of figure 3 , for visual comparison and correlation.

Regarding the comparison of the medical ultrasound data with the velocity data, two aspects can be considered: (1) At the basal layer of the current, close to the flume bottom, there is an obliteration of the perfilometer caused by accumulation of sediments around the velocity probes. This burying effect causes a 
significantly loss in the capacity to measure the effective velocity, which is identified around $20 \mathrm{~s}$. Between 20 and 60s, the burying effect increases as a function of deposition. This region, which lacks effective velocity values, correlates to the abrupt density inversion close to the current bottom (Fig. 4 - B). (2) The highest velocities measured by the perfilometer occur at an intermediate flow region (between 2.5 and $4 \mathrm{~cm}$ ), which is positioned right above the high concentration layer close to the bottom (Fig. 4 - A and B), where the density inversion is identified (Fig $3-\mathrm{A}$ and $\mathrm{B}$ ). The measured velocity at the region close to the bottom is reduced because of the increase in concentration and associated deposition, and that can also be related to the shear stress effect with the bottom interface and flume walls.

\subsection{Treatment of medical ultrasound data to map profile evolution}

For the setup to investigate the physical modeling of a subaqueous slope canyon development resulting from the erosion by turbidity currents, we aimed to document the evolution of the slope profile, which was the focus of the measurements, tracking the angle variations along the slope canyon thalweg. During the experiment, the sensor was kept at the same point, recording the full time evolution. The medical ultrasound data recorded the occurrence of collapses generated by erosion during the passage of the currents.

The selected images (Fig. 5 - A) correspond to: (a) the initial 29을 slope surface, before the turbidity currents reached the area. (b) early erosion and collapse triggered by the passage of the initial turbidity currents; (c) further stage of the profile evolution, caused by retroactive collapses and progressive erosion, where a new equilibrium profile seems to be stablished. (d) final $24^{\circ}$ slope thalweg. This data allowed the construction of an elevation profile graph, delineating a longitudinal section of the slope canyon thalweg (Fig. 5-B).
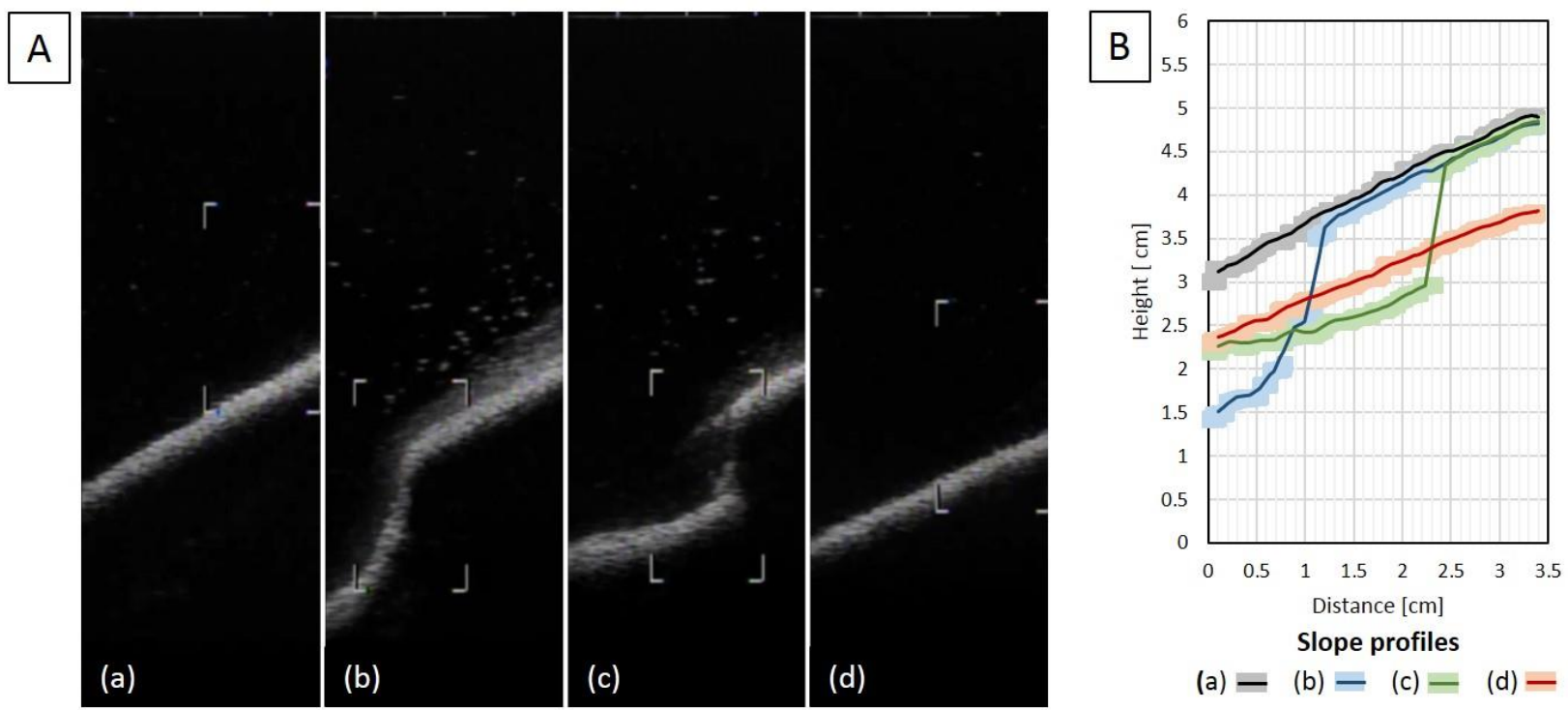

Figure 5. (A) Ultrasound frames captured during the experiment showing the slope profile evolution by the turbidity currents. (B) Bathymetric profiles for different recorded moments. The area analyzed in the ultrasound images is $10 \times 3.5 \mathrm{~cm}$.

\section{DISCUSSION}

The main medical ultrasound feature of a medium for imaging is its impedance contrasts. The impedance depends mainly on density, as it changes the velocity of propagation of the acoustic wave. Considering the studied case, the sound propagation velocity in the medium is a function of its density and the compressibility of the fluids. In the case of the ambient fluid (water) and the modeled turbidity currents, both are considered as incompressible - thus the impedance of the medium would depend only on its density. Although the density is the main parameter, is not the only variable that will change the intensity of the emitted and reflected ultrasound wave. The emitted and received waves undergo an attenuation process, resulting from the sum of the effects of absorption (McDicken \& Anderson, 2011) and (1) dissipation by the mechanical vibration; (2) interference of refracted and diffracted waves; (3) scattering caused by the presence of small particles in the medium and (4) reflection. The scattering phenomenon occurs when the wave strikes particles smaller than the length of the incident wave, usually with the order of 1 to $0.1 \mathrm{~mm}$. The scattering acts reflecting the wave to random directions, decreasing its intensity. The phenomenon of reflection, although it is of interest for the generation of images, also causes important attenuation, since each reflected wave contains a small percentage of the incident wave energy.

The medical ultrasound application for the described experiment measurements, as well as others measuring equipments (i.e., UVP and UHCM) has limited use in high concentrations. The echo received by the transducer is greatly impaired by scattering, as the flow has a large amount of small particles in suspension. 
Among the other attenuation processes, reflection and interference may be the most relevant. In the case of turbidity currents, the concentration profile usually presents an exponential vertical variation, i.e., the attenuation due to the reflection increases exponentially towards the base of the current. It is important to say that our attempts to calibrate the pixel grayscale intensity, attributing a value of density or concentration in each position, were not satisfactory. We are still looking for a better mathematical and experimental formulation to comprehend the attenuation processes.

The data used for the analysis were acquired at different points. The velocity and concentration profilers were positioned 2 meters away from the ultrasound, in the downstream direction. Nevertheless, the visual comparison of the analyzed data, for the interpretation of ultrasound density and the data obtained by the velocity and concentration profilers, presented some correlational similarities. For the experiment with lower concentration (12\%), the intensity inversion zone close to the base of the flow has a thickness similar to the zone identified as representing the increase of concentration and stratification. The region of maximum velocities in this experiment occurs at a position slightly above the basal dense layer (according to UHCM data), yet the ultrasound signal seems not to penetrate this high-density region, as the data in this layer suggests a "low" density by the acquired data. The structures of the upper contour of the mixing layer, identified in the velocity spectrum map are similar to the features identified by the ultrasound, but there is a small delay in the time of occurrence.

Regarding of the geometries identified in the high concentration experiment $(40 \%)$, the thickening of the hyper-concentrated basal layer occurs at a growth rate similar to the abrupt inversion layer of density identified by the ultrasound. In the mixture layer zone, the velocity and concentration profiles do not have a similarity with the ultrasound density profile. The density increase detected was restricted to the zone where the ultrasound sensor was positioned $(4.4 \mathrm{~m})$, and was caused by a greater dilution of the mixture during the injection by the diffuser, not reaching the measurement point of the profilers, located $6.4 \mathrm{~m}$ distant.

The equipment application for surface mapping allowed generating profile erosion curves of the underwater slope. For data processing, even in the images under simultaneous effect of the passage of the turbidity currents, it was possible to visualize the relative position of the surface. The conventional equipment we have in the laboratory works by laser emission. In this case, the presence of suspended sediments perturbs the beam and produces unsatisfactory bathymetric data. The ultrasound is promising because it allows the acquisition of bathymetry data in a continuous way (recording the variation in time), it is not intrusive, and can be used during the experiment, even with the presence of suspended particles.

\section{CONCLUSIONS}

In our experiments with turbidity currents, the medical ultrasound is a useful tool for visualization of flows, sediment transport and erosion of moving substrates. Over time, we have expanded the possibilities of using the equipment, reaching the point of obtaining data capable of producing quantitative information. The use of conventional video cameras, depending of the suspended sediments concentration, do not permit a good visualization of the turbulent structures, internal layers and individual particles. In addition, the conventional video cameras are restricted to observation of the flow with close to the wall (glass surface), being this region under effect of contour interaction.

Another important advantage to use the medical ultrasound, in comparison to others acoustic instruments, is the possibility of use in small-scale experiments (i.e. tanks with $10 \mathrm{~cm}$ wide and $20 \mathrm{~cm}$ wide). The ultrasound is a not intrusive equipment and, in some cases, can be positioned at an adequate distance, not disturbing the flows. However, the equipment requires acoustic dispersers (suspended particles), because in laboratory tests with saline flows (free of suspended particles) there is no good identification and visualization of the flow. As suspended particles act as tracers, the concentration variations along the vertical profile, will project the structures mapped in the modeled flow.

The images analyzed in this study allowed the identification of very similar geometries when comparing ultrasound maps and velocity maps. In ours experiments, which generally use coal as sediment, video camera images do not allow a clear observation of flow geometries and structures. For purpose of comparison, images were acquired by video cameras and medical ultrasound simultaneously, in the same section. This type of record allows important interpretations of the hydrodynamic behavior of the flows, often not so clearly detected using only the velocity and the concentration profilers. The use of the medical ultrasound equipment allowed a mapping of the processes of erosion and deposition during the experiment, promoting a better understanding about the canyon formations.

\section{ACKNOWLEDGEMENTS}

We thank Petrobras (Petróleo Brasileiro S.A.) for the support in the implementation of this experiment, as well as all the support staff of the NECOD laboratory (Núcleo de Estudos em Correntes de Densidade, Universidade Federal do Rio Grande do Sul, Brazil) for the assistance provided in the assembly of the experimental apparatus.

\section{REFERENCES}


Altinakar, M. S. (1988). Weakly depositing turbidity currents on small slopes. PhD thesys à Ecol. Pol. Fed. Lausanne.

Baas, J., Manica, R., Puhl, E., Verhagen, I. \& Borges, A.L. (2014) Processes and products of turbidity currens entering soft muddy substrates. Geology, 42 (5), 371-374.

Best, J., Kirkbride, A. \& Peakall, J. (2001). Mean flow and turbulence structure of sediment-laden gravity currents: new insights using ultrasonic Doppler velocity profiling. Spec. Publ. int. Ass. Sediment., 31, 159172.

Brito, D., Medeiros, D., Manica, R., Borges, A. \& Maestri, R. (2009). Use of visualization equipment (Medical Ultrasound) for the study of non-conservative density currents. Revista Técnica de Energia, Petróleo e Gás, 2, 54-58.( Published in Portuguese).

Chan, V. and Perlas, A. (2011). Basics of Ultrasound Imaging. In: Atlas of Ultrasound-Guided Procedures in Interventional Pain Management. New York, NY: Springer New York. p. 13-19.

Felix, M., Sturton, S. \& Peakall, J. (2005). Combined measurements of velocity and concentration in experimental turbidity currents. Sedimentary Geology, 179, 31-47.

Felix, M. \& Peakall, J. (2006). Transformation of debris flow into turbidity currents: mechanisms inferred from laboratory experiments. Sedimentology, 53, 107-123.

Fildani, A. (2017). Submarine Canyons: A brief review looking forward. Geology, 45(4), 383-383.

Kneller, B. (1995). Beyond the turbidite paradigm: physical models for deposition of turbidites and their implications for reservoir prediction. In: Characterization of deep marine clastic systems. A.J. Hartley (Ed). Pp. 31-49, Geological Society London.

Manica (2009). Generation of high-density turbidity currents: hydraulic and depositional conditioners. Ph.D Thesis. Universidade Federal do Rio Grande do Sul, Brazil. Advisor: Borges, A.L. (Published in Portuguese).

McDicken, W. and Anderson, T. (2011). Basic physics of medical ultrasound. In: Clinical Ultrasound. 2-Volume Set : Expert Consult: Online and Print. Elsevier Health Sciences UK. p. 13-19.

Meiburg, E. \& Kneller, B. (2010). Turbidity currents and their deposits. Annu. Rev. Fluid Mech., 42, 135-156.

Michon, X.; Goddet, J. \& Bonnefille, R. (1955). Etude Theorique et experimentale des courants de densite. 2 vol. Lab. Nat. d'Hydralique Chatou, França.

Paola, C., Straub, K., Mohrig, D. and Reinhardt, L. (2009). The "unreasonable effectiveness" of stratigraphic and geomorphic experiments. Earth-Science Reviews, 97, 1-43.

Paull, C., Ussler, W., Greene, H., Keaten, R., Mitts, P. and Barry, J. (2003). Caught in the act: the 20 December 2001 gravity flow event in Monterey Canyon. Geo-Mar Lett, 22, 227-232.

Sequeiros, O. (2012). Estimating turbidity current conditions from channel morphology: a Froude number approach. Journal of Geophysical Research, 117, C04003.

Talling, P., Masson, D., Sumner, E. and Malgesini, G. (2012). Subaqueous sediment density flows: Depositional process and deposit types. Sedimentology, 59, 1937-2003.

Weimer, P, and Slatt, R. (2004). Petroleum systems of deepwater settings. Society of Exploration Geophysicists, Tulsa, USA, Distinguished Instructor Series, no.7, p.470.

$\mathrm{Xu}, \mathrm{J}$. and Noble, M. (2004). In-situ measurements of velocity structure within turbidity currents. Geophysical Research Letter, 31, L09311.

Xu, J. and Noble, M. (2009). Currents in Monterey Submarine Canyon. Journal of Geophysical Research, 114, C03004.

Xu, J., Sequeiros, O. and Noble, M. (2014). Sediment concentrations, flow conditions, and downstream evolution of two turbidity currents, Monterey Canyon, USA. Deep-Sea Research I, 89, 11-34.

Yalin, M. (1971). Theory of hydraulic models. MacMillan, London, 266 pp. 